\title{
FOLIA
}

Amazónica

Revista del Instituto de Investigaciones

de la Amazonía Peruana

Nota científica

\section{INTRODUCCIÓN Y MANEJO DE PRIMATES EN ISLAS DE LA AMAZONÍA PERUANA - CONSIDERACIONES DE UN POTENCIAL PROBLEMA}

\author{
Eckhard W. HEYMANN ${ }^{1}$ \\ 1 Verhaltensökologie \& Soziobiologie, Deutsches Primatenzentrum - Leibniz-Institut für \\ Primatenforschung, Kellnerweg 4, D-37077 Göttingen, Alemania. Correo electrónico: eheyman@ \\ gwdg.de
}

\section{RESUMEN}

En esta nota se discuten las potenciales consecuencias de una fusión de la Isla Muyuy con la tierra firme, debido a la dinámica fluvial del río Amazonas. La isla alberga una población introducida (alóctona) del pichico de pecho anaranjado, Saguinus labiatus (Callitrichidae). Se recomienda desarrollar un plan de acción, a fin de evitar consecuencias negativas para poblaciones autóctonas de otras especies de pichicos y del ecosistema, en caso que la Isla Muyuy se una con la tierra firme.

PALABRAS CLAVE: Isla Muyuy, Padre Isla, Saguinus labiatus, Saguinus mystax, dinámica fluvial

\section{INTRODUCTION AND MANAGEMENT OF PRIMATES ON ISLANDS IN THE PERUVIAN AMAZON - THOUGHTS ON A POTENTIAL PROBLEM}

\begin{abstract}
This note discusses potential consequences of merging Isla Muyuy on the river Amazon which hosts an introduced (allochthonous) population of red-bellied tamarins, Saguinus labiatus (Callitrichidae), with the mainland, due to the
\end{abstract}


Amazon's riverine dynamics. It is recommended that an action plan be developed for this case, in order to avoid negative consequences for native populations of other species of tamarins and the ecosystem, in case Isla Muyuy joins the mainland.

KEYWORDS: Isla Muyuy, Padre Isla, Saguinus labiatus, Saguinus mystax, riverine dynamics 
La introducción de primates en islas no ocupadas por potenciales competidores y depredadores, es una herramienta de manejo de fauna aplicada por diferentes motivos y en diferentes contextos. En varios lugares de África se introdujeron chimpancés Pan troglodytes en islas, con el fin de rehabilitar individuos que anteriormente vivían en cautiverio (Borner, 1985; Agoramoorthy \& Hsu, 1999). En la Guyana Francesa se introdujeron monos ardilla Saimiri sciureus procedentes de una colonia establecida con fines biomédicos, para evaluar esta opción en la rehabilitación de animales que ya no eran usados en investigaciones o en crianza (Vogel et al., 2002). El éxito de estas introducciones depende mucho del contexto y del esfuerzo invertido (Junker et al., 2017).

En la Amazonía peruana hay dos casos de introducción de primates de la familia Callitrichidae en islas: (1) la introducción de pichicos barba blanca Saguinus mystax mystax, procedentes del lado oriental del río Amazonas (río Ampiyacu, río Manití) a Padre Isla, ubicada a $03^{\circ} 44^{\prime} \mathrm{S} 73^{\circ} 14^{\prime} \mathrm{O}$, cerca de Iquitos (Moya et al., 1990; Gozalo et al., 1991); (2) la introducción de pichicos de pecho anaranjado Saguinus labiatus labiatus, capturados en la zona de Iberia-Iñapari (Madre de Dios) en la Isla Muyuy, ubicada a $03^{\circ} 52^{\prime} \mathrm{S} 73^{\circ} 14^{\prime} \mathrm{O}$ río arriba de Iquitos (Moya et al., 2000; Ique \& Delgado 2016). El éxito de ambas introducciones, medido como supervivencia e incremento de las poblaciones introducidas, posiblemente se debe a dos factores principales: (1) los animales introducidos procedían del medio natural y de esta manera estaban habilitados para buscar sus alimentos y evitar riesgos como p. ej. depredadores; (2) además de los alimentos disponibles en el hábitat, se les proporcionó alimentos adicionales, al menos en el caso de Padre Isla. Además, Padre Isla se convirtió en un lugar donde se realizaron estudios ecológicos que, en combinación con estudios del hábitat natural, permiten evaluar la adaptabilidad y flexibilidad de los pichicos (Gazzo, 1990; Heymann, 1990; Garber et al., 1993; Garber, 1998; Tornow et al., 2006).

El presente trabajo busca llamar la atención sobre el riesgo potencial existente en la introducción de primates en las islas. Cuando una isla se junta con la tierra firme, los primates que fueron introducidos colonizan el nuevo hábitat, alterando la fauna autóctona de ese lugar.

En la Amazonía, al igual que en otras regiones del mundo, los ríos, sobre todo aquellos de gran tamaño, pueden formar barreras geográficas que afectan a la distribución de diferentes organismos (Patton et al., 1994, 2000; Gascon et al., 2000). No obstante, por regla general los ríos no representan barreras absolutas, existiendo cierta permeabilidad que depende de la dinámica fluvial y de la movilidad de los animales. Un factor en la dinámica fluvial es la formación de islas ocasionada por la ruptura de ríos meándricos y la posterior fusión de estas islas con la tierra firme al lado opuesto del río. De esta manera, puede ocurrir una transferencia pasiva de organismos de un lado al otro del río (en contraste con la dispersión activa ocasionada por organismos que cruzan una barrera por propio movimiento). Hershkovitz (1983) presentó este escenario para explicar la presencia del mono nocturno Aotus nancymaae en un enclave al norte del río Marañón. De la misma manera, Peres et al. (1996) explicaron la presencia de híbridos entre dos especies/subespecies de pichicos del género Leontocebus (que incluye todos los taxa [especies y subespecies] anteriormente considerados como Saguinus fuscicollis y Saguinus nigricollis; véase Rylands et al., 2016) en el curso medio y alto del río Juruá debido la dinámica fluvial. La dinámica fluvial es muy común (Ward et al., 2002; Jackson \& Austin, 2013) y ha sido documentada para el río Amazonas, p. ej. por Salo et al. (1986) y García Sánchez (1987). 
En el caso de Padre Isla, una fusión con el lado oriental del río Amazonas no representaría un problema, porque los $S$. mystax de Padre Isla proceden del lado oriental. Una fusión con el lado occidental no es probable, dado que allá se encuentra el puerto de Iquitos. En el caso de la Isla Muyuy, la situación es muy diferente. La isla puede unirse al lado oriental o al occidental del río Amazonas, en cualquiera de los dos casos, los $S$. labiatus no proceden de ninguna de estas zonas, por lo que indistintamente invadirían un nuevo hábitat. Este escenario podría tener tres potenciales resultados: (1) los $S$. labiatus no son capaces de adaptarse al nuevo hábitat y la población se extingue, este escenario no es muy probable, ya que los $S$. labiatus se han adaptado muy bien a las condiciones de la Isla Muyuy; (2) los S. labiatus colonizan e invaden exitosamente el nuevo hábitat y desplazan a otras especies de pichicos, como S. mystax y Leontocebus nigrifrons en el lado oriental y Leontocebus lagonotus en el lado occidental del río Amazonas; (3) los $S$. labiatus colonizan e invaden exitosamente el nuevo hábitat e hibridan con $S$. mystax en el lado oriental del río Amazonas, algo que ya ha sido reportado en especies hermanas de Saguinus en estado de cautividad (Hershkovitz, 1977).

Desde la perspectiva de la conservación, los escenarios (2) y (3) no son deseables. La invasión de hábitats por especies alóctonas puede crear diversos problemas, desde la extinción de especies autóctonas, hasta una serie de interferencias con el funcionamiento de los ecosistemas (Pejchar \& Mooney, 2009). Un ejemplo pertinente para la probable extinción de especies autóctonas debido a la invasión de especies alóctonas, es el de los titíes (familia Callitrichidae) Callithrix aurita y Callithrix flaviceps (ambos autóctonos) y Callithrix jacchus y Callithrix penicillata (ambos alóctonos) en el suroriente de Brasil (Pereira et al., 2008; Detogne et al., 2017; de Carvalho et al., 2018; Malukiewicz, 2019). Las poblaciones de $C$. aurita y $C$. flaviceps han sido desplazadas de sus zonas de distribución por poblaciones de $C$. jacchus y $C$. penicillata, que son producto de la introducción intencional del ser humano o la huida de gran número de individuos de su cautiverio. El desplazamiento se realiza por competencia directa y por hibridación y puede reducir en un futuro las poblaciones de las especies autóctonas en un 50\% (Melo et al., 2015 citado en Detogne et al., 2017; Nunes, 2015 citado en Detogne et al., 2017; Malukiewicz, 2019). A nivel mundial, la hibridación entre especies autóctonas y especies introducidas representa un problema de conservación, afectando poblaciones tanto de animales como de plantas (Rhymer \& Simberloff, 1996; Allendorf et al., 2001). La introducción del ánade real, Anas platyrhynchos, está afectando la existencia de poblaciones autóctonas de otras especies de ánades en Hawaii, Florida y Nueva Zelanda (Rhymer \& Simberloff, 1996). El aumento de poblaciones de gatos domésticos y la hibridización con el gato silvestre, Felis silvestris, es una amenaza para este último en Escocia (Hubbard et al., 1992). La introducción de macacos de cola de cerdo Macaca nemestrina, pone en riesgo las últimas poblaciones de macacos rheso Macaca mulatta en Tailandia (Malaivijitnond et al., 2007). La mayoría de los ejemplos provienen de las zonas templadas, posiblemente debido a los mayores esfuerzos de investigación realizados. No obstante, estos ejemplos y, en particular, el de los Callithrix, deberían servir de alerta para el escenario posible en el que la Isla Muyuy se fusiona con la tierra firme. Dada la dinámica del río Amazonas, esto podría ocurrir en un futuro lejano, pero también en uno cercano. Para evitar consecuencias negativas, sería apropiado desarrollar un plan de acción para este escenario posible (Bohling, 2016; Jackiw et al., 2015). 


\section{REFERENCIAS BIBLIOGRÁFICAS}

Agoramoorthy, G.; Hsu, M.J. 1999. Rehabilitation and release of chimpanzees on a natural island. Methods hold promises for other primates as well. Journal of Wildlife Rehabilitation, 22: 3-7.

Allendorf, F.W.; Leary, R.F.; Spruell, P.; Wenburg, J.K. 2001. The problems with hybrids: setting conservation guidelines. Trends in Ecology \& Evolution, 16: 613-622. DOI: https://doi. org/10.1016/S0169-5347(01)02290-X

Bohling, J.H. 2016. Strategies to address the conservation threats posed by hybridization and genetic introgression. Biological Conservation, 203: 321-327. DOI: https://doi. org/10.1016/j.biocon.2016.10.011

Borner, M. 1985. The rehabilitated chimpanzees of RubondoIsland.Oryx,19:151-154.DOI:https:// doi.org/10.1017/S003060530002531X

de Carvalho, R.S.; Bergallo, H.G.; Cronemberger, C.; Guimarães-Luiz, T.; Igayara-Souza, C.A.; Jerusalinsky, L.; Knogge, C.; Lacerda, W.R.; Malukiewicz, J.; de Melo, F.R.; Moreira, S.B.; Pereira, D.G.; Pissinatti, A.; Port-Carvalho, M.; Ruiz-Miranda, C.R.; Wormell, D. 2018. Callithrix aurita: a marmoset species on its way to extinction in the Brazilian Atlantic Forest. Neotropical Primates, 24: 1-8.

Detogne, N.; Ferreguetti, Á.C.; Mello, J.H.F.; Santana, M.C.; da Conceição Dias, A.; da Mota, N.C.J.; Esteves da Cruz Gonçalves, A.; de Souza, C.P.; Bergallo, H.G. 2017. Spatial distribution of buffy-tufted-ear (Callithrix aurita) and invasive marmosets (Callithrix spp.) in a tropical rainforest reserve in southeastern Brazil. American Journal of Primatology, 79: e22718. DOI: https://doi.org/10.1002/ajp.22718

Garber, P.A. 1998. Within-and between-site variability in moustached tamarin (Saguinus mystax) positional behavior during food procurement. En: Strasser, E.; Fleagle, J.G.; Rosenberger, A.L.; McHenry, H.M. (Eds).
Primate Locomotion: Recent Advances. Plenum Press, New York. p. 61-78.

Garber, P.A.; Pruetz, J.D.; Isaacson, J. 1993. Patterns of range use, range defense, and intergroup spacing in moustached tamarin monkeys (Saguinus mystax). Primates, 34: 11-25. DOI: https://doi.org/10.1007/BF02381276

García Sánchez, J. 1987. El río que se aleja. Cambio del curso del Amazonas. Historia y estudio técnico. CETA, Iquitos.

Gascon, C.; Malcolm, J.R.; Patton, J.L.; da Silva, M.N.F.; Bogart, J.P.; Lougheed, S.C.; Peres, C.A.; Necckel, S.; Boag, P.T. 2000. Riverine barriers and the geographic distribution of Amazonian species. Proceedings of the National Academy of Sciences of the USA, 97: 13672-13677. DOI: https://doi.org/10.1073/pnas.230136397

Gazzo, C. 1990. Estudios bioecológicos de tres grupos del pichico barba blanca (Saguinus mystax Spix 1823) en Padre Isla, Iquitos. In: Castro-Rodríguez, N.E. (Ed). La Primatología en el Perú. Proyecto Peruano de Primatología "Manuel Moro Sommo". Investigaciones Primatológica (1973-1985). Imprenta Propaceb., Lima. p. 506-525.

Gozalo, A.; Moya, L.; Ique, C.; Moro, J.; Encarnación, F. 1991. Breeding the moustached tamarin (Saguinus mystax) on islands. In: Ehara, A. (Ed). Primatology Today. Elsevier Science Publishers, Amsterdam. p. 401-402.

Hershkovitz, P. 1977. Living New World monkeys (Platyrrhini) 1. University of Chicago Press, Chicago.

Hershkovitz, P. 1983. Two new species of night monkeys, genus Aotus (Cebidae, Platyrrhini): a preliminary report on Aotus taxonomy. American Journal of Primatology, 4: 209-243. DOI: https://doi.org/10.1002/ ajp.1350040302

Heymann, E.W. 1990. Social behaviour and infant carrying in a group of moustached tamarins, Saguinus mystax (Primates: Platyrrhini: 
Callitrichidae), on Padre Isla, Peruvian Amazonia. Primates, 31: 183-196. DOI: https:// doi.org/10.1007/BF02380940

Hubbard, A. L., McOrist, S., Jones, T. W., Boid, R., Scott, R., Easterbee, N. (1992). Is survival of European wildcats Felis silvestris in Britain threatened by interbreeding with domestic cats?. Biological Conservation, 61: 203-208.

Ique, C., Delgado, A. 2016. Dinámica poblacional de Saguinus labiatus labiatus (Geoffroy in Humboldt, 1812) en Isla Muyuy, río Amazonas, Loreto, Perú. Conocimiento Amazónico, 7: 119125.

Jackiw, R.N.; Mandil, G.; Hager, H.A. 2015. A framework to guide the conservation of species hybrids based on ethical and ecological considerations. Conservation Biology, 29: 1040-1051. DOI: https://doi.org/10.1111/ cobi. 12526

Jackson, N.D.; Austin, C.C. 2013. Testing the role of meander cutoff in promoting gene flow across a riverine barrier in ground skinks (Scincella lateralis). PLoS ONE, 8: e62812-e62812. DOI: $\quad$ https://doi.org/10.1371/journal. pone.0062812

Junker, J.; Kühl, H.S.; Orth, L.; Smith, R.K.; Petrovan, S.O.; Sutherland, W.J. (2017). Primate

conservation: Global evidence for the effects of interventions. Cambridge: University of Cambridge.

Malaivijitnond, S.; Takenaka, O.; Kawamoto, Y.; Urasopon, N.; Hadi, I.; Hamada, Y. 2007. Anthropogenic macaque hybridization and genetic pollution of a threatened population. Natural History Journal of Chulalongkorn University, 7: 11-23.

Malukiewicz, J. 2019. A review of experimental, natural, and anthropogenic hybridization in Callithrix marmosets. International Journal of Primatology, 40: 72-98. DOI: https://doi. org/10.1007/s10764-018-0068-0

Moya, L. ; Ique, C. ; Soini, M. 1990. Introducción experimental y desarollo de una población de Saguinus mystax en Padre Isla, Iquitos, Perú. En: Castro-Rodríguez, N.E. (Ed.) La Primatología en el Perú. Proyecto Peruano de Primatología "Manuel Moro Sommo". Investigaciones Primatológica (1973-1985). Imprenta Propaceb, Lima. p. 526-536.

Moya, L.; Ique, C.; Puertas, P.; Encarnación, F. 2000. Liberación de los "pichico pecho anaranjado" (Saguinus labiatus) en la Isla de Muyuy. En: Felipe San Martín, H.F.; Marco García, P. (Eds). La Primatología en el Perú II. Proyecto Peruano de Primatología "Manuel Moro Sommo". Master Graf Editores, Lima. p. 109-120.

Patton, J.L.; da Silva, M.N.F; Malcolm, J.R. 1994. Gene genealogy and differentiation among arboreal spiny rats (Rodentia: Echimyidae) of the Amazon basin: a test of the riverine barrier hypothesis. Evolution, 48: 1314-1323. DOI: https://doi.org/10.1111/j.1558-5646.1994. tb05315.x

Patton, J.L.; da Silva, M.N.F.; Malcolm, J.R. 2000. Mammals of the Rio Juruá and the evolutionary and ecological diversification of Amazonia. Bulletin of the American Museum of Natural History, 244: 1-306. DOI: https://doi. org/10.1206/0003-0090(2000)244\%3C0001 :MOTRJA\%3E2.0.CO;2

Pejchar, L.; Mooney, H.A. 2009. Invasive species, ecosystem services and human well-being. Trends in Ecology \& Evolution, 24: 497-504. DOI: https://doi.org/10.1016/j.tree.2009.03.016

Pereira, D.G.; Oliveira, M.E.A.; Ruiz-Miranda, C.R. 2008. Interações entre calitriquídeos exóticos e nativos no Parque Nacional da Serra dos Órgãos - RJ. Revista Espaço e Geografia, 11: 87114.

Peres, C.A.; Patton, J.L.; da Silva, M.N.F. 1996. Riverine barriers and gene flow in Amazonian saddle-back tamarins. Folia Primatologica, 67: 113-124. DOI: https://doi. org/10.1159/000157213 
Rhymer, J. M.; Simberloff, D. 1996. Extinction by hybridization and introgression. Annual Review of Ecology and Systematics, 27: 83109. DOI: https://doi.org/10.1146/annurev. ecolsys.27.1.83

Rylands, A.B.; Heymann, E.W.; Lynch Alfaro, J.; Buckner, J.C.; Roos, C.; Matauschek, C.; Boubli, J.P.; Sampaio, R.; Mittermeier, R.A. 2016. Taxonomic review of the New World tamarins (Primates: Callitrichidae). Zoological Journal of the Linnean Society, 177: 1003-1028. DOI: https://doi.org/10.1111/zoj.12386

Salo, J.; Kalliola, R.; Hakkinen, I. 1986. River dynamics and the diversity of Amazon lowland forest. Nature, 322: 254-258. DOI: https://doi. org/10.1038/322254a0
Tornow, M.A.; Ford, S.M.; Garber, P.A. ; de sa Sauerbrunn, E. 2006. Dentition of moustached tamarins (Saguinus mystax mystax) from Padre Isla, Peru, part 1: quantitative variation. American Journal of Physical Anthropology, 130: 352-363. DOI: https://doi.org/10.1002/ ajpa. 20374

Vogel, I.; Glöwing, B.; Saint Pierre, I.; Bayart, F.; Contamin, H. ; de Thoisy, B. 2002. Squirrel monkey (Saimiri scireus) rehabilitation in French Guiana: a case study. Neotropical Primates, 10: 147-149.

Ward, J.V.; Tockner, K.; Arscott, D.B.; Claret, C. 2002. Riverine landscape diversity. Freshwater Biology, 47: 517-539. DOI: https://doi. org/10.1046/j.1365-2427.2002.00893.x

Recibido: 4 de febrero de 2020 Aceptado para publicación: 2 de marzo de 2020

Esta obra está bajo una Licencia Creative Commons Atribución-NoComercial-SinDerivar 4.0 Internacional. 